Research Article

\title{
Fetal Rhesus D Genotyping and Sex Determination from Maternal Plasma of Rhesus D-Negative Antenatal Population: The Usefulness of Conventional Polymerase Chain Reaction in Resource-limited Settings
}

\author{
Otchere Addai-Mensah ${ }^{\mathbb{D}},{ }^{1}$ Edward Y. Afriyie, ${ }^{1}$ Samuel Asamoah Sakyi $\mathbb{D}^{\mathbb{D}}{ }^{2}$ \\ Christian Obirikorang, ${ }^{2}$ Max Efui Annani-Akollor, ${ }^{1}$ Eddie-Williams Owiredu (D, ${ }^{1}$ \\ Francis A. Amponsah, ${ }^{1}$ Richard Vikpebah Duneeh, ${ }^{3}$ and Evans Asamoah Adu ${ }^{1}{ }^{1}$ \\ ${ }^{1}$ Department of Medical Diagnostics, Faculty of Allied Health Sciences, College of Health Sciences, \\ Kwame Nkrumah University of Science and Technology, Kumasi, Ghana \\ ${ }^{2}$ Department of Molecular Medicine, School of Medical Science, Kwame Nkrumah University of Science and Technology, \\ Kumasi, Ghana \\ ${ }^{3}$ Department of Medical Laboratory Sciences, School of Allied Health Sciences, University of Health and Allied Sciences, \\ Ho, Ghana
}

Correspondence should be addressed to Otchere Addai-Mensah; drmedmozart@yahoo.com

Received 28 May 2020; Revised 19 September 2020; Accepted 6 October 2020; Published 16 October 2020

Academic Editor: George Uchenna Eleje

Copyright $\odot 2020$ Otchere Addai-Mensah et al. This is an open access article distributed under the Creative Commons Attribution License, which permits unrestricted use, distribution, and reproduction in any medium, provided the original work is properly cited.

\begin{abstract}
Background. This prospective cohort study evaluated the usefulness of conventional PCR in genotyping fetal Rhesus D (RhD) and sex from the maternal plasma of $\mathrm{RhD}$-negative ( $\mathrm{RhD}-$ ) antenatal population in resource-limited settings. Methods. Thirty apparently healthy $\mathrm{RhD}$ - pregnant women with $\mathrm{RhD}$ positive $(\mathrm{RhD}+)$ partners were included. Blood samples were collected from each participant (in the third trimester of pregnancy) for DNA extraction/purification and fetal RhD genotyping. Results. Out of the 30 samples, 26 (86.7\%) were found to be $\mathrm{RhD}+$ while 4 (13.3\%) were $\mathrm{RhD}-$. The $\mathrm{RhD}+$ comprised $24(80.0 \%) \mathrm{RhD}+$ based on exons 5, 7, and 10 combined. Exons 5 and 7 were detected in two additional samples but not exon 10. Serological phenotyping of neonatal blood confirmed $26 \mathrm{RhD}+$ and $4 \mathrm{RhD}-$. There was a perfect agreement between the fetal $\mathrm{RhD}$ genotype and neonatal $\mathrm{RhD}$ phenotyping after delivery for exons 5 and 7 (concordance $=100 \%, \kappa=100.0 \%$, diagnostic accuracy $=100 \%, p<0.0001$ ) while exon 10 presented with an almost perfect agreement (concordance $=93.3 \%, \kappa=76.2 \%$, diagnostic accuracy $=93.3 \%$, $p<0.0001)$. Regarding the prenatal test for the SRY gene, $9(30.0 \%)$ were predicted to be males and the remaining $21(60.0 \%)$ were females. All the 9 and 21 anticipated males and females, respectively, were confirmed after delivery (concordance $=100 \%$, $\kappa=100.0 \%$, diagnostic accuracy $=100 \%)$. Conclusion. Our study suggests that conventional PCR using the SRY, RhD exons 5 and 7 could be useful for predicting fetal sex and $\mathrm{RhD}$ from maternal peripheral blood in resource-limited settings.
\end{abstract}

\section{Background}

The Rhesus (Rh) blood group system is one of the most polymorphic and immunogenic systems known in humans. In the past decade, extensive investigations have yielded considerable knowledge about the molecular background of this system [1]. The antigens of the $\mathrm{Rh}(\mathrm{RhAg})$ blood group system, namely, D, C, c, E, and e are encoded by the homologous genes RHD and RHCE, both located on chromosome 1 [1].

An RhD-negative phenotype primarily occurs as a result of the deletion of the entire RHD gene. Rh antibodies ( $\mathrm{RhAb}$ ) are formed due to the exposure of an Rh-negative mother to cells which bears the RhAg, commonly through transfusions 
or pregnancy $[2,3]$. These Abs have been implicated in Hemolytic Transfusion Reactions, Autoimmune Hemolytic Anemia, and Hemolytic Disease of the Fetus and Newborn (HDFN) [4]. HDFN is an important cause of perinatal mortality and morbidity arising from transplacental passage of maternal $\mathrm{RhD} \mathrm{Ab}$, mostly IgG, their binding to fetal erythrocyte $\mathrm{RhD} \mathrm{Ag}$, and the subsequent destruction of the fetal erythrocytes $[5,6]$. This underscored the need for preemptive strategies against fetal haemolysis and subsequent fetal loss.

The introduction of anti-D prophylaxis has drastically reduced the global incidence of $\mathrm{RhD}$ immunization from $14 \%$ to $0.8-1.5 \%$, with some countries having as low as $0.2-0.4 \%$ [7]. Nonetheless, the use of anti-D prophylaxis is not without risks (such as infections) as Rh immunoglobulin is derived from pooled human plasma $[3,8]$. There is thus the need for effort intensification to avoid unessential exposure through the confirmation of fetal $\mathrm{RhD}$ status.

Advances in $\mathrm{RhD}$ genotyping technology have made it possible to noninvasively determine fetal $\mathrm{RhD}$ from analysis of circulating cell-free fetal DNA (ccffDNA) in maternal plasma as against amniocentesis $[6,9,10]$. ccffDNA originates from apoptotic macrovesicles separated from trophoblast cells of the placenta $[11,12]$. It circulates in maternal blood at low levels detectable from the $7^{\text {th }}$ week of gestation-its level increases proportionally with increasing gestational age until it diminishes and disappears 1-2 days after delivery [13]. The advent of robotic systems of fetal DNA isolation has also made it possible for high-throughput testing [14-16]. However, the cost involved in using these systems may be too huge for most developing countries to defray. Recently, the use of real-time polymerase chain reaction ( $\mathrm{qPCR}$ ) has gained considerable attention in its usefulness in ascertaining fetal sex and $\mathrm{RhD}$ genotype [17-19]. Nevertheless, this method is not only expensive and requires relatively high expertise but also unavailable in most developing countries.

Unlike developed countries where prenatal $\mathrm{RhD}$ genotyping is considered routine and prophylaxis given only after genotype is determined, many developing countries embrace the practice of administering anti-D immunoglobulin prophylaxis to all $\mathrm{RhD}$-negative pregnant women with $\mathrm{RhD}$ positive husbands. Rationalization for this practice can be ascribed to the possibility of bearing an $\mathrm{RhD}$ positive fetus as well as the lack of sophisticated equipment to prenatally determine fetal RhD status. There is however an indication that a significant proportion of $\mathrm{RhD}$-negative women carry RhD-negative fetus [20]; thus, there is a possibility that anti$\mathrm{D}$ immunoglobulin could be administered superfluously to a substantial number of women in such instances.

This highlights the need for developing countries to determine fetal RhD genotype to ensure that anti-D immunoglobulin is purposefully administered to the right candidate $[21,22]$. Additional merit for fetal $\mathrm{RhD}$ genotyping is that it precludes the cost burden of anti-D immunoglobulin as well as the risks associated with the administration of blood products $[10,23]$.

This study evaluated the usefulness of the comparatively less expensive conventional PCR for fetal sex and RhD genotyping at a teaching hospital in Kumasi, Ghana. The findings of this study will equip policymakers with the necessary information that would inform the inclusion of fetal RhD genotyping as routine testing during pregnancy in developing countries with limited resources.

\section{Methods}

2.1. Study Design and Participants. This was a prospective cohort study conducted at the Komfo Anokye Teaching Hospital (KATH), Kumasi, Ghana. The duration of the study was one year: between January 2017 and 2018. Thirty (30) apparently healthy RhD-negative pregnant women with RhD positive partners attending the antenatal clinic were purposively recruited. Only participants who consented after the study objectives were explained to them were included in the study.

2.2. Ethical Considerations. Ethical approval for this study was obtained from the Committee on Human Research, Publications and Ethics (CHRPE), School of Medical Sciences, Kwame Nkrumah University of Science \& Technology (Ref: CHRPE/RC/252/17). Written informed consent was obtained from all participants who opted to participate. Participation was voluntary, and respondents were assured that the information obtained was strictly for research and academic purposes only and were guaranteed the liberty to opt out of the study at their own convenience.

2.3. Sample Collection and Preparation. Five milliliters ( $5 \mathrm{ml})$ of venous blood was collected from each participant into EDTA tubes and spun at $2000 \mathrm{~g}$ for 10 minutes to obtain the plasma which was transferred into fresh tubes using filter tips and recentrifuged at $3000 \mathrm{~g}$ for 10 minutes. The supernatant was separated and stored at $-80^{\circ} \mathrm{C}$ until further processing. All samples were processed within 6 hours of sample collection.

2.4. DNA Extraction/Purification and Genotyping. DNA was extracted using the standard modified inorganic (salting out) method as previously described by Miller et al. [24]. Briefly, cell lysis was performed by suspending the sample in lysis buffer (155 mM NH $4 \mathrm{Cl}, 10 \mathrm{mM} \mathrm{NH}_{4} \mathrm{CO}_{3}, 1 \mathrm{mM}$ EDTA), followed by incubation on ice and centrifugation at $3,000 \mathrm{rpm}$ and $4^{\circ} \mathrm{C}$ for $30 \mathrm{~min}$. The pellets were resuspended in TE buffer and centrifuged to obtain white blood cell pellets which were resuspended in nucleic lysis buffer

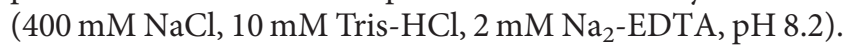
Cell lysates were digested with proteinase $\mathrm{K}$ and $10 \%$ sodium dodecyl sulfate (SDS) followed by the addition of $6 \mathrm{M} \mathrm{NaCl}$ and nucleic lysis buffer and centrifugation at 4,600 rpm for $60 \mathrm{~min}$. DNA precipitation was performed by the addition of cold $96 \%$ ethanol. The quantities of the isolated DNA were measured using the NanoDrop 3300 Fluorospectrometer (Thermo Fisher Scientific Inc., USA) and A260/280 of 1.8-2.0 was used to define DNA purity (Supplemental Material: Figure S1). Each sample was assessed for fetal RhD 
and sex genotype by conventional PCR as previously described [25]. Briefly, thirty cycles of denaturation for $1 \mathrm{~min}$ at $92^{\circ} \mathrm{C}$, primer annealing for $1 \mathrm{~min}$ at $49^{\circ} \mathrm{C}$, and primer extension for $1 \mathrm{~min}$ at $72^{\circ} \mathrm{C}$ were performed, followed by a final extension for $9 \mathrm{~min}$ at $72^{\circ} \mathrm{C}$. The reaction products were separated by agarose gel electrophoresis (3\% agarose gel with $0.5 \mu \mathrm{g} / \mathrm{ml}$ of ethidium bromide) and visualized under ultraviolet light (Supplemental Material: Figure S2). In an effort to enhance the specificity of the assay, we included three RHD gene regions, namely, RhD exons 5, 7, and 10. ccffDNA was confirmed using $\mathrm{Y}$ chromosome specific primer SRY. The primers for fetal SRY, RhD exons 5, 7, and 10 are shown in Supplemental Material: Table S1.

2.5. Confirmation of the Fetal Sex and RhD Status. Sex neonates were ascertained at birth. Furthermore, peripheral blood samples were collected immediately after birth from each neonate and $\mathrm{RhD}$ phenotype determined based on serological blood grouping (Diahem Diagnostics, Switzerland).

2.6. Statistical Analysis. Continuous and categorical data were presented as median (interquartile range) and frequency (percentages), respectively. Chi-square test and Cohen's Kappa were used to assess the association and concordance between prenatal and postnatal measures. A $p$ value $<0.05$ was considered statistically significant. Statistical analysis was performed using IBM SPSS version 25.0.

\section{Results}

The average maternal and gestational ages of the study participants were 28.0 years and 33 weeks, respectively. A higher proportion were in their third trimester $(80.0 \%)$, were married (73.3\%), and had secondary education (53.3\%) (Table 1).

Out of the 30 maternal samples analyzed for fetal $\mathrm{RhD}$ genotype, 26 (86.7\%) were detected as RhD positive while 4 (13.3\%) were detected as $\mathrm{RhD}$-negative. The $\mathrm{RhD}$ positives comprised a total of $24(80.0 \%) \mathrm{RhD}$ positives based on $\mathrm{RhD}$ exons 5,7 , and 10 combined. RhD exons 5 and 7 were detected in two additional samples $(2 / 6.6 \%)$ but not $\mathrm{RhD}$ (exon 10) (Table 2). Serological phenotyping of neonatal blood confirmed $26 \mathrm{RhD}$ positives (86.7\%) and 4 (13.3\%) $\mathrm{RhD}$ negatives. There was perfect agreement ( concordance $=100 \%, \quad \kappa=100.0 \%, \quad$ diagnostic accuracy $=100 \%$ ) between fetal $\mathrm{RhD}$ genotyping and neonatal $\mathrm{RhD}$ phenotyping after delivery for exons 5 and 7 while exon 10 presented with almost perfect agreement (concordance $=93.3 \%, \quad \kappa=76.2 \%, \quad$ diagnostic accuracy $=93.3 \%)($ Table 3$)$.

Regarding the prenatal test of the SRY gene, 9 (30.0\%) of the cases were predicted to be males and the remaining 21 (60.0\%) females (Table 2). All 9 and 21 anticipated males and females, respectively, were confirmed after delivery (concordance $=100 \%, \quad \kappa=100.0 \%, \quad$ diagnostic accuracy $=100 \%)($ Table 3$)$.
TABle 1: Demographic and obstetric characteristics of the study participants.

\begin{tabular}{lcc}
\hline Variable & Frequency $(n=30)$ & Percentage (\%) \\
\hline Maternal age (years) & $28.0(25.0-31.0)^{*}$ & \\
$\quad<25$ & 5 & 16.7 \\
$25-30$ & 17 & 56.7 \\
$31-35$ & 8 & 26.7 \\
$>35$ & & \\
\hline Gestational age (weeks) & $33.0(12.0-39.0)^{*}$ & \\
$\quad$ First & 2 & 6.7 \\
Second & 4 & 13.3 \\
Third & 24 & 80 \\
Parity & $1(0-2)^{*}$ & \\
Gravidity & $2(1-3)^{*}$ & \\
\hline Marital status & & \\
$\quad$ Married & 22 & 23.3 \\
Single & 8 & 3.3 \\
\hline Educational status & & 23.3 \\
$\quad$ No formal education & 1 & 53.3 \\
Basic & 7 & 20 \\
Secondary & 16 & \\
Tertiary & 6 & \\
* Data are presented as median (interquartile range).
\end{tabular}

\section{Discussion}

Most developed countries perform prenatal $\mathrm{RhD}$ genotyping and prophylaxis is given only after fetal $\mathrm{RhD}$ positive genotype in an $\mathrm{RhD}$-negative mother is determined. The robotic systems [14-16] as well as qPCR are utilized in these countries for highly accurate fetal $\mathrm{RhD}$ genotyping $[17,18]$. However, these methods are not only expensive but also unavailable in most developing countries. For this reason, many healthcare facilities in developing countries embrace the practice of administering anti-D immunoglobulin prophylaxis to all $\mathrm{RhD}$-negative pregnant women with $\mathrm{RhD}$ positive partners irrespective of fetus $\mathrm{RhD}$ status. Though useful as there have been drastic reductions in the incidence of $\mathrm{RhD}$ immunization since the inception of the anti-D immunoglobulin prophylaxis, the disadvantages associated with unsighted administration of the prophylaxis underpin the need for prenatal fetal $\mathrm{RhD}$ genotyping. This study evaluated the expediency of the less expensive conventional PCR for fetal sex and RhD genotyping in resource-limited settings.

Of the 30 maternal samples investigated, 26 (86.7\%) were $\mathrm{RhD}$ positive while $4(13.3 \%)$ were $\mathrm{RhD}$ negative. This suggests that over $13 \%$ of the pregnant women attending antenatal care will untenably receive anti-D immunoprophylaxis if fetal $\mathrm{RhD}$ genotype is not determined. Though the number of patients associated with this proportion is low given the limited sample size of this study, considering the increasing number of pregnancies in developing countries $[26,27]$, this number could be potentially high. Thus, putting measures in place to abate the unessential administration of anti-D immunoprophylaxis will be of tremendous benefit to both the mother and her unborn baby.

In assessing the expediency of conventional PCR for prenatal sex and $\mathrm{RhD}$ genotyping for use in resource-limited 
TABLE 2: Fetal sex, RhD genotype from maternal plasma, and their confirmation after delivery.

\begin{tabular}{|c|c|c|c|c|c|c|}
\hline \multirow{2}{*}{ Patient ID } & \multicolumn{4}{|c|}{ Fetal genotype from maternal blood } & \multirow{2}{*}{ Neonatal RhD phenotype after delivery } & \multirow{2}{*}{ Confirmed sex of neonates } \\
\hline & RHD 5 & RHD 7 & RHD 10 & SRY & & \\
\hline 1 & + & + & + & - & + & Female \\
\hline 2 & + & + & + & - & + & Female \\
\hline 3 & + & + & - & + & + & Male \\
\hline 4 & + & + & + & - & + & Female \\
\hline 5 & - & - & - & - & - & Female \\
\hline 6 & + & + & + & - & + & Female \\
\hline 7 & + & + & + & + & + & Male \\
\hline 8 & + & + & + & + & + & Male \\
\hline 9 & + & + & + & - & + & Female \\
\hline 10 & + & + & + & - & + & Female \\
\hline 11 & - & - & - & - & - & Female \\
\hline 12 & + & + & + & - & + & Female \\
\hline 13 & + & + & + & + & + & Male \\
\hline 14 & + & + & + & - & + & Female \\
\hline 15 & + & + & + & - & + & Female \\
\hline 16 & + & + & + & - & + & Female \\
\hline 17 & + & + & + & - & + & Female \\
\hline 18 & - & - & - & + & - & Male \\
\hline 19 & + & + & + & - & + & Female \\
\hline 20 & + & + & + & + & + & Male \\
\hline 21 & + & + & + & + & + & Male \\
\hline 22 & + & + & - & - & + & Female \\
\hline 23 & + & + & + & - & + & Female \\
\hline 24 & + & + & + & - & + & Female \\
\hline 25 & + & + & + & - & + & Female \\
\hline 26 & + & + & + & + & + & Male \\
\hline 27 & - & - & - & - & - & Female \\
\hline 28 & + & + & + & + & + & Male \\
\hline 29 & + & + & + & - & + & Female \\
\hline 30 & + & + & + & - & + & Female \\
\hline
\end{tabular}

+; positive, -; negative.

TABLE 3: Analytical and diagnostic measures between prenatal RhD and sex genotyping and postnatal measures.

\begin{tabular}{|c|c|c|c|c|c|c|}
\hline \multirow{2}{*}{ Genotyping } & \multicolumn{2}{|c|}{ Neonatal RhD phenotype } & \multirow{2}{*}{ Concordance } & \multirow{2}{*}{ Cohen's kappa } & \multirow{2}{*}{ Accuracy } & \multirow{2}{*}{$P$ value } \\
\hline & $\mathrm{RhD}+$ & RhD- & & & & \\
\hline \multicolumn{7}{|c|}{ Fetal genotype } \\
\hline \multicolumn{7}{|c|}{$\mathrm{RhD5}$} \\
\hline Positive & $26(100.0)$ & $0(0.0)$ & $100.0 \%$ & $100.0 \%$ & $100.0 \%$ & $<0.0001$ \\
\hline Negative & $0(0.0)$ & $4(100.0)$ & & & & \\
\hline \multicolumn{7}{|l|}{ RhD7 } \\
\hline Positive & $26(100.0)$ & $0(0.0)$ & $100.00 \%$ & $100.0 \%$ & $100.0 \%$ & $<0.0001$ \\
\hline Negative & $0(0.0)$ & $4(100.0)$ & & & & \\
\hline \multicolumn{7}{|l|}{ RhD10 } \\
\hline Positive & $24(92.3)$ & $0(0.0)$ & $92.30 \%$ & $76.2 \%$ & $93.3 \%$ & 0.001 \\
\hline Negative & $2(7.7)$ & $4(100.0)$ & & & & \\
\hline Fetal gender & \multicolumn{6}{|c|}{ Neonatal gender } \\
\hline $\begin{array}{l}\text { SRY } \\
\end{array}$ & Male & Female & & & & \\
\hline Positive & $9(100.0)$ & $0(0.0)$ & $100 \%$ & $100.00 \%$ & $100.00 \%$ & $<0.0001$ \\
\hline Negative & $0(0.0)$ & $21(100.0)$ & & & & \\
\hline
\end{tabular}

facilities, we found that neonates of $\mathrm{RhD}$ - pregnant women with $\mathrm{RhD}+$ partners can be genotyped with an excellent level of accuracy (100\%) for SRY, RhD exons 5 and 7 but not RhD 10 which showed a $93.3 \%$ accuracy. Specifically, comparing prenatal evaluations with postnatal assessments, fetal RhD exons 5 and 7 genotyping from maternal plasma with a median gestational age of 33.0 weeks showed a $100 \%$ concordance with neonatal $\mathrm{RhD}$ phenotypes after delivery. This finding is consistent with a study by Minon et al. [6] who found that by combining amplification of three exons, the concordance rate of fetal $\mathrm{RhD}$ genotypes in maternal plasma and newborn D phenotypes at delivery was $100 \%(99.8 \%$ 
including one unusual false-positive) based on qPCR. Also in harmony with our study findings are the studies by Lo et al. [15] and Hromadnikova et al. [28]. Evidence suggests that, in order to avoid false-negative results, at least two exons of RHD need to be assessed [20,29]. Thus, according to the findings of this study, conventional PCR employing the SRY, RhD exons 5 and 7 may be valuable for genotyping fetal sex and $\mathrm{RhD}$ from maternal peripheral blood in resource-limited settings which do not possess the sophisticated real-time PCR. A recent study by Mahdavi et al. also confirms the usefulness of the SRY gene for fetal sex determination [19].

The major limitation of this study is that we did not perform qPCR in tandem with the conventional PCR for comparison. However, this limitation is attenuated due to the fact that the prospective nature of the study sex and fetal $\mathrm{RhD}$ status were confirmed after the babies were born. Furthermore, the study involved a relatively small sample size. We thus recommend the use of a larger sample size in future studies.

\section{Conclusion}

Our study suggests that conventional PCR using the SRY, $\mathrm{RhD}$ exons 5 and 7 could be useful for predicting fetal sex and $\mathrm{RhD}$ from maternal peripheral blood in resource-limited settings.[30]

\section{Data Availability}

All relevant data are within the article (and its supplementary information files).

\section{Conflicts of Interest}

The authors declare no conflicts of interest.

\section{Acknowledgments}

The authors are grateful to the Staff of the Komfo Anokye Teaching Hospital, Kumasi, Ghana, and all who actively participated in the study.

\section{Supplementary Materials}

The primers for SYR, RhD exons 5, 7, and 10 used in this study (table S1), graph of DNA purity (figure S1), and yield and agarose gel electrophoresis as visualized under ultraviolet light are given (figure S2). (Supplementary Materials)

\section{References}

[1] N. D. Avent and M. E. Reid, "The Rh blood group system: a review," Blood, vol. 95, no. 2, pp. 375-387, 2000.

[2] L. Cardo, B. P. García, and F. V. Alvarez, "Non-invasive fetal RHD genotyping in the first trimester of pregnancy," Clinical Chemistry and Laboratory Medicine, vol. 48, pp. 1121-1126, 2010.

[3] G. M. Liumbruno, A. D'alessandro, F. Rea et al., "The role of antenatal immunoprophylaxis in the prevention of maternal- foetal anti-Rh (D) alloimmunisation," Blood Transfusion, vol. 8, no. 1, pp. 8-16, 2010.

[4] K.-H. Kim, K.-E. Kim, K.-S. Woo, J.-Y. Han, J.-M. Kim, and K. U. Park, "Primary anti-D immunization by DEL red blood cells," Annals of Laboratory Medicine, vol. 29, no. 4, pp. 361-365, 2009.

[5] C. Rouillac-Le Sciellour, P. Puillandre, R. Gillot et al., "Largescale pre-diagnosis study of fetal RHD genotyping by PCR on plasma DNA from RhD-negative pregnant women," Molecular Diagnosis, vol. 8, no. 1, pp. 23-31, 2004.

[6] J. M. Minon, C. Gerard, J. M. Senterre, J. P. Schaaps, and J. M. Foidart, "Routine fetal RHD genotyping with maternal plasma: a four-year experience in Belgium," Transfusion, vol. 48, no. 2, pp. 373-381, 2008.

[7] A. T. Wikman, E. Tiblad, A. Karlsson, M. L. Olsson, M. Westgren, and M. Reilly, "Noninvasive single-exon fetal RHD determination in a routine screening program in early pregnancy," Obstetrics \& Gynecology, vol. 120, no. 2 Pt 1, pp. 227-234, 2012.

[8] B. M. Kumpel, "Lessons learnt from many years of experience using anti-D in humans for prevention of RhD immunization and haemolytic disease of the fetus and newborn," Clinical \& Experimental Immunology, vol. 154, no. 1, pp. 1-5, 2008.

[9] N. D. Avent, "RHD genotyping from maternal plasma: guidelines and technical challenges," in Prenatal Diagnosis, pp. 185-201, Springer, Salmon, NY, USA, 2008.

[10] M. Grande, E. Ordoñez, V. Cirigliano et al., "Clinical application of midtrimester non-invasive fetal RHD genotyping and identification of RHD variants in a mixed-ethnic population," Prenatal Diagnosis, vol. 33, no. 2, pp. 173-178, 2013.

[11] E. Flori, B. Doray, E. Gautier et al., "Circulating cell-free fetal DNA in maternal serum appears to originate from cyto- and syncytio-trophoblastic cells. Case report," Human Reproduction, vol. 19, no. 3, pp. 723-724, 2004.

[12] H. Masuzaki, K. Miura, K. Yoshiura, S. Yoshimura, N. Niikawa, and T. Ishimaru, "Detection of cell free placental DNA in maternal plasma: direct evidence from three cases of confined placental mosaicism," Journal of Medical Genetics, vol. 41, no. 4, pp. 289-292, 2004.

[13] L. Birch, C. A. English, K. O’Donoghue, O. Barigye, N. M. Fisk, and J. T. Keer, "Accurate and robust quantification of circulating fetal and total DNA in maternal plasma from 5 to 41 weeks of gestation," Clinical Chemistry, vol. 51, no. 2, pp. 312-320, 2005.

[14] J. T. Aubin, C. Le Van Kim, I. Mouro et al., "Specificity and sensitivity of RHD genotyping methods by PCR-based DNA amplification," British Journal of Haematology, vol. 98, no. 2, pp. 356-364, 1997.

[15] Y. M. D. Lo, N. M. Hjelm, C. Fidler et al., "Prenatal diagnosis of fetal RhD status by molecular analysis of maternal plasma," New England Journal of Medicine, vol. 339, no. 24, pp. 1734-1738, 1998.

[16] R. Akolekar, K. Finning, R. Kuppusamy, G. Daniels, and K. H. Nicolaides, "Fetal," Fetal Diagnosis and Therapy, vol. 29, pp. 301-306, 2011.

[17] I. N. Machado, L. Castilho, J. Pellegrino, and R. Barini, "Fetal rhd genotyping from maternal plasma in a population with a highly diverse ethnic background," Revista da Associação Médica Brasileira, vol. 52, pp. 232-235, 2006.

[18] M. H. Ahmadi, S. Hantuoshzadeh, M. A. Okhovat, N. Nasiri, A. Azarkeivan, and N. Amirizadeh, "Fetal RHD genotyping from circulating cell-free fetal DNA in plasma of rh negative pregnant women in Iran," Indian Journal of Hematology and Blood Transfusion, vol. 32, pp. 447-453, 2016. 
[19] S. Mahdavi, F. Karami, and S. Sabbaghi, "Non-invasive prenatal diagnosis of foetal gender through maternal circulation in first trimester of pregnancy," Journal of Obstetrics and Gynaecology, vol. 39, no. 8, pp. 1-4, 2019.

[20] C. E. Van Der Schoot, G. M. Tax, R. J. Rijnders, M. De Haas, and G. C. Christiaens, "Prenatal typing of Rh and Kell blood group system antigens: the edge of a watershed," Transfusion Medicine Reviews, vol. 17, pp. 31-44, 2003.

[21] D. R. Amaral, D. C. Credidio, J. Pellegrino, and L. Castilho, "Fetal RHD genotyping by analysis of maternal plasma in a mixed population," Journal of Clinical Laboratory Analysis, vol. 25, pp. 100-104, 2011.

[22] F. Banch Clausen, "Integration of noninvasive prenatal prediction of fetal blood group into clinical prenatal care," Prenatal Diagnosis, vol. 34, pp. 409-415, 2014.

[23] Y.-J. Zhu, Y.-R. Zheng, L. Li et al., "Diagnostic accuracy of non-invasive fetal RhD genotyping using cell-free fetal DNA: a meta analysis," The Journal of Maternal-Fetal \& Neonatal Medicine, vol. 27, pp. 1839-1844, 2014.

[24] S. Miller, D. Dykes, and H. Polesky, "A simple salting out procedure for extracting DNA from human nucleated cells," Nucleic Acids Research, vol. 16, p. 1215, 1988.

[25] S. N. El Din, A. R. Aramy, and M. S. Ali, "Correlation between the $\mathrm{RhD}$ genotyping and $\mathrm{RhD}$ serotyping in isoimmunized pregnancies," Egyptian Journal of Medical Human Genetics, vol. 12, pp. 127-133, 2011.

[26] A. Kott, "Rates of unintended pregnancy remain high in developing regions," International Perspectives on Sexual and Reproductive Health, vol. 37, no. 1, pp. 46-47, 2011.

[27] J. Bearak, A. Popinchalk, L. Alkema, and G. Sedgh, "Global, regional, and subregional trends in unintended pregnancy and its outcomes from 1990 to 2014: estimates from a Bayesian hierarchical model," The Lancet Global Health, vol. 6, no. 4, pp. e380-e389, 2018.

[28] I. Hromadnikova, L. Vechetova, K. Vesela, B. Benesova, J. Doucha, and R. Vlk, "Non-invasive fetal RHD and RHCE genotyping using real-time PCR testing of maternal plasma in RhD-negative pregnancies," Journal of Histochemistry \& Cytochemistry, vol. 53, no. 3, pp. 301-305, 2005.

[29] G. Daniels, K. Finning, P. Martin, and E. Massey, "Noninvasive prenatal diagnosis of fetal blood group phenotypes: current practice and future prospects," Prenatal Diagnosis: Published in Affiliation with the International Society for Prenatal Diagnosis, vol. 29, no. 2, pp. 101-107, 2009.

[30] C. E. Van Der Schoot, S. Hahn, and L. S. Chitty, "Non-invasive prenatal diagnosis and determination of fetal Rh statused," Seminars in Fetal and Neonatal Medicine Elsevier, vol. 13, no. 2, pp. 63-68, 2008. 